\title{
COVID-19 and myeloproliferative neoplasms: some considerations
}

\author{
Baransel Kamaz $\mathbb{D}^{1} \cdot$ Ann Mullally $\mathbb{D}^{1}$
}

Received: 20 August 2020 / Revised: 16 September 2020 / Accepted: 13 October 2020 / Published online: 26 October 2020

(c) Springer Nature Limited 2020

\section{To the Editor,}

COVID-19 cases are rising daily around the globe. Although, patients with hematological cancers have been reported to have a higher case fatality rate [1], clinical data for patients with BCR-ABL negative myeloproliferative neoplasms (MPN) who developed COVID-19 are currently lacking. In thinking about the potential impact of COVID19 in MPN, we have considered three main areas:

\section{(1) MPN disease-intrinsic factors.}

MPN patients have been reported to demonstrate NK cell dysfunction [2] and T-cell exhaustion [3], even in the absence of treatment. This dysregulation in cellular immunity observed in MPN patients could potentially result in an impaired antiviral response to SARS-CoV-2 infection. Inflammatory monocytes also contribute to the dysregulated immune system in MPN patients via cytokine overproduction [4], which could potentially cause a deleterious inflammatory response upon SARS-CoV-2 infection.

MPN is characterized by a predisposition to thrombosis, which is a consequence of both quantitative and qualitative abnormalities in myeloid blood cells [5]. COVID-19 is associated with a high prevalence of coagulopathy and thrombosis [6]. Although the mechanisms underlying COVID19-associated coagulopathy remain an area of active investigation, it appears that thrombo-inflammation resulting in increased procoagulant factor levels (e.g., fibrinogen) and endothelial cell damage both play significant roles [6]. Neutrophil extracellular trap formation has also been linked to

Ann Mullally

amullally@partners.org

1 Division of Hematology, Department of Medicine, Brigham and Women's Hospital, Harvard Medical School, Boston, MA, USA
COVID-19-related thrombosis [7], as well as to MPN-related thrombosis [8].

(2) MPN therapy.

The JAK1/2 inhibitor ruxolitinib, FDA-approved for the treatment of myelofibrosis and polycythemia vera (PV), inhibits JAK-STAT signaling in myeloid blood cells, but also has suppressive effects in NK cells [2] and $\mathrm{T}$ cells, resulting in its recent approval for the treatment of steroid-refractory-acute-graftversus-host disease [9]. Consequently, one could hypothesize that in patients with MPN currently receiving ruxolitinib, SARS-CoV-2 infection may be more severe due to suppressed cellular immunity. Intriguingly, due to its broad anti-inflammatory effects, ruxolitinib is under active investigation for the treatment of patients with COVID-19 (who do not have MPN), initially in a pilot study [10] and currently in multiple clinical trials (see Table 1). For patients with MPN who are receiving ruxolitinib at the time of SARS-CoV-2 infection, it remains to be determined whether this proves beneficial in counteracting the inflammatory phase of COVID-19.

Interferon alpha (IFNa), which is used to treat essential thrombocythemia and PV, has broad antiviral activity and is also under investigation in COVID-19 patients in clinical trials (see Table 1). Based on previous experience in the setting of Middle East respiratory syndrome coronavirus infection [11], the timing of initiation of IFNa treatment relative to the time of SARS-CoV-2 infection is likely to be important, with early treatment having the potential to decrease viral replication, while late treatment could potentially enhance the proinflammatory environment and exacerbate disease. Hydroxyurea is the most common MPN drug prescribed in the U.S. Based on clinical observations and patient data analysis, initiating hydroxyurea therapy for stroke prevention in sickle-cell anemia during the COVID-19 pandemic has been suggested [12]. Currently, there are no clinical trials for the use of hydroxyurea in COVID-19. 


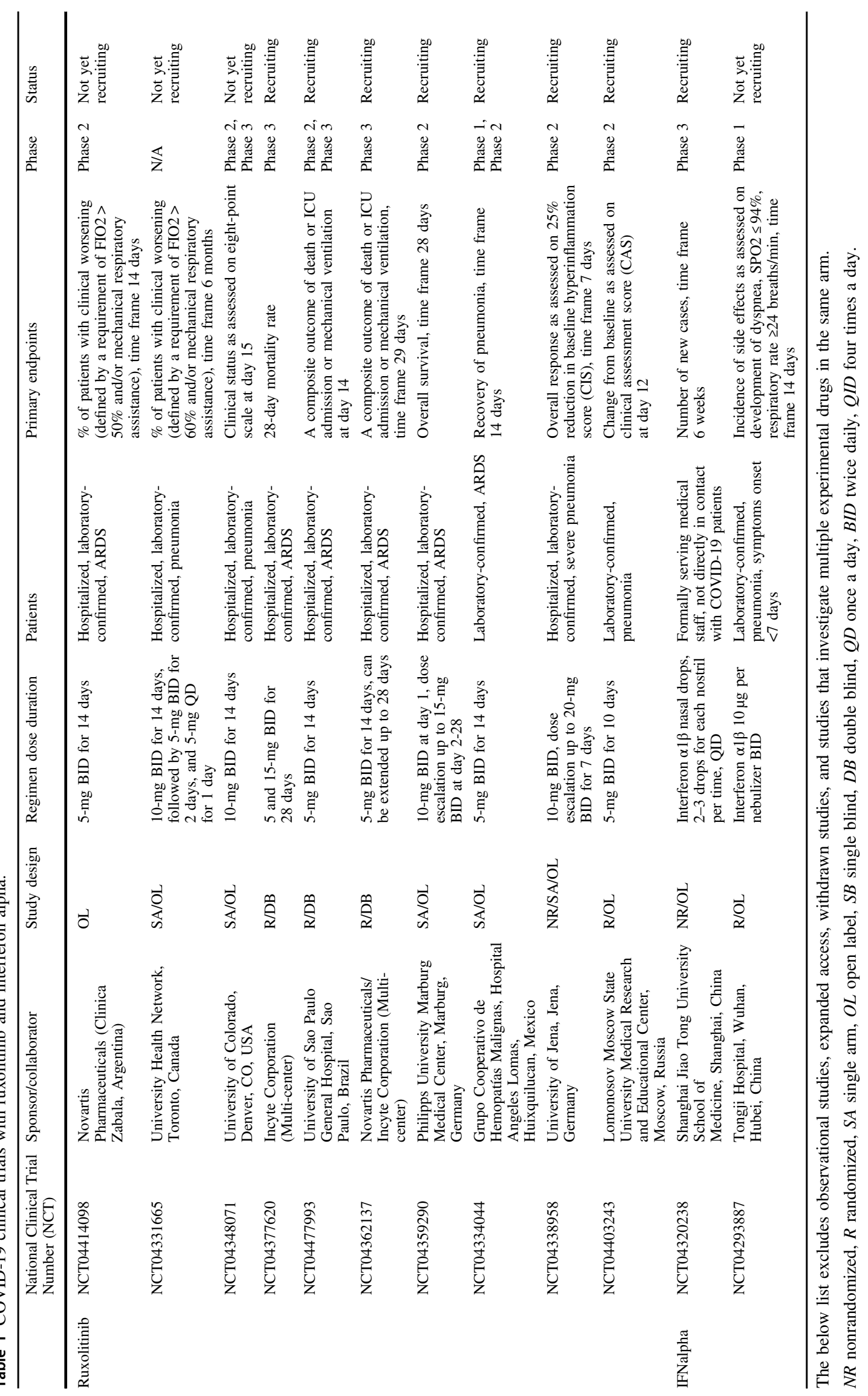


(3) Clinical considerations in treating COVID-19 in the context of MPN.

MPN is characterized by an inflammatory state [13], the impact of which in the setting of COVID-19 is yet to be elucidated. Given the clinical and molecular heterogeneity of MPN, studying outcomes in MPN patients who develop COVID-19 will inform future treatment algorithms. Currently, the COVID-19 Treatment Guidelines Panel recommends against the use of JAK inhibitors and IFNa for COVID-19 except in clinical trials, due to the broad immunosuppressive effects and lack of efficacy in previous coronavirus infections, respectively [14]. MPN patients are known to have increased thrombosis risk, and this risk is reduced through the use of cytoreductive therapy (e.g., hydroxyurea) in high-risk patients. Based on current recommendations, COVID-19 should not change the algorithm for venous thrombo-embolism (VTE) prophylaxis per the standard of care for hospitalized adults [14]. However, this is an area of active clinical investigation and recommendations for VTE prophylaxis in hospitalized patients with COVID-19 (including those with MPN) are in evolution. In terms of MPN patients, it is not advised to change MPN-directed therapy in patients who develop COVID-19, (e.g., abruptly discontinuing ruxolitinib could result in ruxolitinib withdrawal syndrome [15], which has the potential to be more severe in the setting of COVID19). Another consideration is the phase of SARS-CoV2 infection and the specific MPN treatment e.g., MPN patients on IFNa could potentially have an advantage early in the course of COVID-19 and potentially a disadvantage later in the course of disease. For ruxolitinib, the opposite could potentially be the case; disadvantageous early in COVID-19 and potentially advantageous later.

In summary, there are many aspects of MPN and its treatment that are particularly relevant in the midst of the COVID-19 pandemic. We hope that the considerations we have outlined here will help MPN physicians be attune to these issues as we await clinical data from MPN patients with COVID-19 to emerge.

Acknowledgements This work was supported by the NIH (R01HL131835 to AM), the MPN Research Foundation (AM), and the Gabrielle's Angel Foundation for Cancer Research (AM). AM is a Scholar of The Leukemia \& Lymphoma Society. We thank Dr Jean Connors for reviewing the manuscript.

Author contributions $\mathrm{BK}$ and $\mathrm{AM}$ designed the outline for the manuscript. BK drafted the manuscript. BK and AM edited and approved the manuscript.

\section{Compliance with ethical standards}

Conflict of interest AM has received honoraria from Blueprint Medicines, Roche, and Incyte for invited lectures and receives research support from Janssen and Actuate Therapeutics.

Publisher's note Springer Nature remains neutral with regard to jurisdictional claims in published maps and institutional affiliations.

\section{References}

1. He W, Chen L, Chen L, Yuan G, Fang Y, Chen W, et al. COVID-19 in persons with haematological cancers. Leukemia. 2020;34:1637-45.

2. Schönberg K, Rudolph J, Vonnahme M, Parampalli Yajnanarayana S, Cornez I, Hejazi M, et al. JAK inhibition impairs NK cell function in myeloproliferative neoplasms. Cancer Res. 2015;75:2187-99.

3. Cimen Bozkus C, Roudko V, Finnigan JP, Mascarenhas J, Hoffman $\mathrm{R}$, Iancu-Rubin $\mathrm{C}$, et al. Immune checkpoint blockade enhances shared neoantigen-induced T-cell immunity directed against mutated calreticulin in myeloproliferative neoplasms. Cancer Disco. 2019; 9:1192-207.

4. Fisher DAC, Miner CA, Engle EK, Hu H, Collins TB, Zhou A, et al. Cytokine production in myelofibrosis exhibits differential responsiveness to JAK-STAT, MAP kinase, and NFKB signaling. Leukemia. 2019;33:1978-95.

5. Stein BL, Martin K. From Budd-Chiari syndrome to acquired von Willebrand syndrome: thrombosis and bleeding complications in the myeloproliferative neoplasms. Hematol Am Soc Hematol Educ Program. 2019;2019:397-406.

6. Connors JM, Levy JH. COVID-19 and its implications for thrombosis and anticoagulation. Blood. 2020;135:2033-40.

7. Zuo Y, Yalavarthi S, Shi H, Gockman K, Zuo M, Madison JA, et al. Neutrophil extracellular traps in COVID-19. JCI Insight. 2020;5:e138999.

8. Wolach O, Sellar RS, Martinod K, Cherpokova D, McConkey M, Chappell RJ, et al. Increased neutrophil extracellular trap formation promotes thrombosis in myeloproliferative neoplasms. Sci Transl Med. 2018;10:eaan8292.

9. Zeiser R, von Bubnoff N, Butler J, Mohty M, Niederwieser D, Or $\mathrm{R}$, et al. Ruxolitinib for glucocorticoid-refractory acute graftversus-host disease. N Engl J Med. 2020;382:1800-10.

10. La Rosée F, Bremer HC, Gehrke I, Kehr A, Hochhaus A, Birndt S, et al. The Janus kinase $1 / 2$ inhibitor ruxolitinib in COVID-19 with severe systemic hyperinflammation. Leukemia. 2020;34:1805-15.

11. Channappanavar R, Fehr AR, Zheng J, Wohlford-Lenane C, Abrahante JE, Mack M, et al. IFN-I response timing relative to virus replication determines MERS coronavirus infection outcomes. J Clin Invest. 2019;129:3625-39.

12. DeBaun MR. Initiating adjunct low-dose hydroxyurea therapy for stroke prevention in children with SCA during the COVID-19 pandemic. Blood. 2020;135:1997-9.

13. Koschmieder S, Mughal TI, Hasselbalch HC, Barosi G, Valent P, Kiladjian JJ, et al. Myeloproliferative neoplasms and inflammation: whether to target the malignant clone or the inflammatory process or both. Leukemia. 2016;30:1018-24.

14. NIH. COVID-19 treatment guidelines. 2020. https://covid19trea tmentguidelines.nih.gov/.

15. Coltro G, Mannelli F, Guglielmelli P, Pacilli A, Bosi A, Vannucchi AM. A life-threatening ruxolitinib discontinuation syndrome. Am J Hematol. 2017;92:833-8. 